\title{
Aspectos cinemáticos del caballo criollo argentino al trote
}

\author{
Kinematic aspects of the criollo argentino horse \\ at trot
}

\section{Aspectos cinemáticos do cavalo criole argentino na trota}

\author{
Verna $E^{1}$, Audisio $S A^{1}$, Vaquero $P^{1}$, Rossetto $L^{1}$, Arriagada $V D^{1}$, Milanta $G^{1}$ \\ 1 Facultad de Ciencias Veterinarias, Universidad Nacional de La Pampa. Cátedra Técnica y Patología \\ Quirúrgica. Calle 5 esqu. 116 General Pico. La Pampa. República Argentina.
}

Correo electrónico:saudisio@vet.unlpam.edu.ar

Fecha de recepción: 10/02/2021

Fecha aceptación para su publicación: 22/07/2021

"En memoria del Dr. Edgardo Verna"

\section{RESUMEN}

Los estudios biocinemáticos se emplean con fines clínicos, fisioterápicos y en programas de reproducción. El objetivo de este estudio fue aportar información de estas variables para el caballo Criollo Argentino. Se estudiaron 8 yeguas de la raza Criollo Argentino. Les fueron colocados marcadores refractantes en sitios anatómicos predeterminados. Se les hizo recorrer una distancia de 15 metros sobre césped guiadas de la mano. Los recorridos se repitieron tres veces por individuo y se registraron en video. Los fotogramas se introdujeron en el software ImageJ para medir los ángulos articulares, de inclinación escapular y pélvica, protracción y retracción y de extensión, flexión y amplitud angular de cada articulación de los miembros, a la vez que se estableció la longitud y número de zancadas por batida y la velocidad a la que recorrieron los 15 metros. Se realizó estadística descriptiva de las variables. Los caballos recorrieron la distancia en 22,99 seg a 
4,08 m/seg para lo cual ejecutaron 5,69 zancadas con una longitud de batida de 2,66 m efectuada en 0,65 seg. Los ángulos de protracción y retracción fueron $22,63^{\circ}$ y $6,8^{\circ} ; 9,06^{\circ}$ y $22,03^{\circ}$ para miembros torácico y pélvico. Los ángulos de inclinación escapular y pelviano fueron

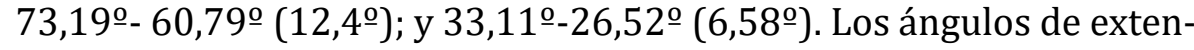
sión y flexión y amplitud angular fueron: hombro 128,07-116,93을 $11,13^{\circ}$, codo $159,85^{\circ}-144,60^{\circ}, 15,52^{\circ}$; carpo $175,85^{\circ}-172,18^{\circ}, 3,66^{\circ}$, cadera $103,38^{\circ}-86,19$ ㅇ $, 17,18^{\circ}$; rodilla $138,7^{\circ}-118,56^{\circ}, 20,01^{\circ}$; tarso

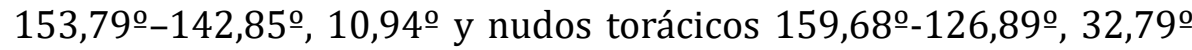
y pelviano $161,09^{\circ}-129,68^{\circ}, 31,41^{\circ}$. Los rangos de retracción-protracción de los miembros contribuyen a conocer la sincronicidad de la retracción ipsilateral de la extremidad anterior y la protracción del miembro posterior en el trote. La ejecución del trote en el caballo Criollo Argentino requiere sincronización de extensiones y flexiones articulares cuyas variaciones mínimas y máximas fueron registradas.

Palabras clave: Caballo Criollo Argentino, articulación, ángulo, cinemática

\section{SUMMARY}

Biokinematic variables of the locomotion of the Criollo Argentino breed of horses, of which there is no specific information. These variables may be used with clinical and physiotherapeutic objectives and in reproduction programmes. 8 Criollo Argentino mares were used in the study. Refractive markers were placed in anatomical sites previously determined. They were made to trot covering a distance of 15 meters on grass guided by hand. Each individual repeated the activity three times, which were recorded on video. The photograms were introduced in the software ImageJ to measure the joint angles of scapular and pelvic inclination, protraction, retraction, extension, flexion and angular amplitude of each joint in the limbs. Simultaneously, the length and number of strides for all limbs and the speed at which they covered 15 meters. The statistical description of variables was carried out. The horses covered the distance in $22.99 \mathrm{sec}$. at $4.08 \mathrm{~m} / \mathrm{sec}$. doing 5.69 strides of a length of $2.66 \mathrm{~m}$. per $0.65 \mathrm{sec}$. The protraction and retraction angles were $22.63^{\circ}$ and $6.8^{\circ} ; 9.06^{\circ}$ and $22.03^{\circ}$ for the thoracic and pelvic limbs. The scapular and pelvic inclination angles were

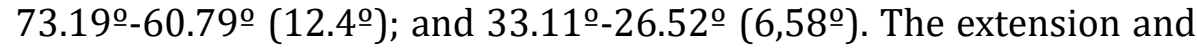
flexion angles and angular amplitude were: shoulder $12807^{\circ}-116.93^{\circ}$, $11.13^{\circ}$, elbow $159.85^{\circ}-144.60^{\circ}$, $15.52^{\circ}$; carpus $175.85^{\circ}-172.18^{\circ}$, $3.66^{\circ}$, hip $103.38^{\circ}-86.19^{\circ}$, $17.18^{\circ}$; p patella $138.57^{\circ}-118.56^{\circ}$, $20.01^{\circ}$; tarsus 
$153.79^{\circ}-142.85^{\circ}, 10.94^{\circ}$ and thoracic limb fetlock $159.68^{\circ}-126.89^{\circ}$, $32.79^{\circ}$ and pelvic limb fetlock $161.09^{\circ}-129.68^{\circ}$, 31.41ㅇ. The range of retraction-protraction of the limbs contributes to the knowledge of synchronicity of the ipsilateral retraction of the forelimb and the protraction of the hindlimb during trotting. The Criollo Argentino's performance at trot requires the synchronization of joint extension and flexion whose minimal and maximum variations were registered.

Key words: Criollo Argentino horse, joint, angle, kinematic

\section{RESUMO}

Os estudos biocinemáticos são usados para programas clínicos, fisioterapêuticos e reprodutivos. $\mathrm{O}$ objetivo deste estudo foi fornecer informações sobre essas variáveis para o cavalo Crioulo argentino. Foram estudadas 8 éguas da raça Crioula Argentino. Os marcadores refrativos foram colocados em locais anatômicos predeterminados. Eles foram obrigados a percorrer uma distância de 15 metros na grama guiados de mãos dadas. Os passeios foram repetidos três vezes por indivíduo e gravados em vídeo. Os quadros foram inseridos no software ImageJ para medir os ângulos articulares, de inclinação escapular e pélvica, protração e retração e de extensão, flexão e amplitude angular de cada articulação dos membros, ao mesmo tempo que o comprimento e número de passadas eram por batimento e a velocidade com que percorreram os 15 metros. Foi realizada estatística descritiva das variáveis. Os cavalos cobriram a distância em 22,99 seg a 4,08 m / seg, para a qual executaram 5,69 passadas com um comprimento de passada de 2,66 m realizado em 0,65 seg. Os ângu-

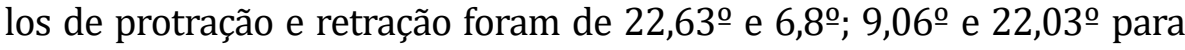
membros torácicos e pélvicos. Os ângulos de inclinação escapular e pélvico foram $73,19^{\circ}-60,79^{\circ}\left(12,4^{\circ}\right)$; e $33,11^{\circ}-26,52^{\circ}\left(6,58^{\circ}\right)$. Os ângulos de extensão e flexão e amplitude angular foram: ombro $128,07^{\circ}-116,93^{\circ}$, $11,13^{\circ}$, cotovelo $159,85^{\circ}-144,60^{\circ}$, $15,52^{\circ}$; carpo $175,85^{\circ}-172,18^{\circ}$, 3,66은,

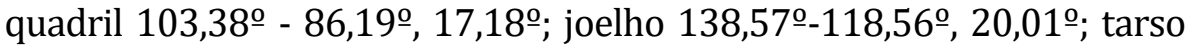

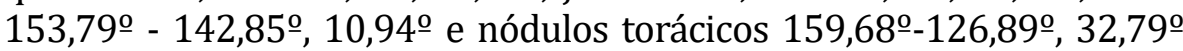
e nós pélvicos $161,09^{\circ}-129,68^{\circ}, 31,41^{\circ}$. Os intervalos de retração-protração dos membros contribuem para o conhecimento da sincronicidade da retração ipsilateral do membro anterior e da protração do membro posterior no trote. A execução do trote no cavalo Crioulo argentino requer a sincronização das extensões e flexões articulares cujas variações mínimas e máximas foram registradas.

Palavras chave: Cavalo Crioulo Argentino, junta, ângulo, cinemática 


\section{Introducción}

Los estudios biocinemáticos aportan información cuantitativa de los patrones normales de la marcha. Brindan conocimientos de las fuerzas actuantes sobre los miembros, contribuyen a diagnosticar lesiones músculo esqueléticas, asisten a la evaluación de tratamientos y constituyen un método válido para comprender las adaptaciones de la locomoción siguiendo patrones durante la marcha ${ }^{[1-5]}$.

Ciertos parámetros biocinemáticos como la frecuencia de batidas, actividad dorsoventral y actividad longitudinal que poseen heredabilidad media a alta, constituyen variables de selección genética en programas de cría. Incluso el producto de las cruzas entre razas que al momento de ejecutar distintos andares indican que poseen sustento genético, ya que la cruza entre ejemplares de razas óptimas para distintas actividades brinda descendencia adecuada. Por su parte, Valera y col. ${ }^{[7]}$ en estudios de la raza Pura Española establecieron las variables lineales, temporales y angulares biocinemáticas y la correlación que poseen de heredabilidad.

Con los métodos biocinemáticos se pueden obtener los parámetros de desplazamiento de velocidad, aceleración lineal, ángulo de flexión de las articulaciones, velocidad angular y aceleración angular de los segmentos durante un andar u otra actividad como el salto ${ }^{[7]}$. Las características cinemáticas se hallan descriptas para las razas empleadas en competencias de trote ${ }^{[3,8,10]}$, razas Árabes y Español ${ }^{[11]}$, Pura Sangre de Carrera ${ }^{[12]}$, caballos de Silla ${ }^{[13]}$, Chilote de Paso Fino ${ }^{[14]}$, raza Bardgiano $^{[15]}$, caballo de Menorca ${ }^{[16]}$; caballo Andaluz ${ }^{[17]}$, Islandés ${ }^{[18]} y$ puro Español ${ }^{[3]}$. Sin embargo, en la raza Criolla no se ha encontrado estudios específicos opublicados para estandarizar. El objetivo del presente trabajo consistió en establecer parámetros biocinemáticos del caballo de la raza Criollo Argentino para ser aplicados en el diagnóstico de afecciones locomotoras y efectuar seguimiento de tratamientos de enfermedades del aparato locomotor.

\section{Materiales y métodos}

Se emplearon 8 yeguas de la raza Criollo Argentino puras de raza registradas en la Asociación de Criadores de Caballos Criollo Argentino, cuya altura a la cruz osciló entre 150,5 y 164,8 cm, procedentes de una cabaña inscripta en la Asociación de Criadores de Caballos Criollos, ubicada en el Departamento Maracó, La Pampa, Argentina. 
Antes de iniciar el estudio, cada equino fue examinado para descartar dolencias del aparato locomotor que pudieran desvirtuar la información obtenida.

A cada yegua le fueron colocados marcadores reflectantes de 3,20 cm de diámetro, adheridos con pegamento de contacto a la piel de los miembros torácicos y pelvianos izquierdos. En los miembros torácicos los marcadores se situaron en la piel que cubre el punto más elevado de la cruz, tuberosidad de la espina escapular (tuber spina scapulae), porción caudal de la tuberosidad mayor del húmero (tuberculum major humerus (pars caudalis), ligamento colateral cubital lateral (lig. collaterale cubiti laterale) proceso estiloideo lateral del radio (procesus styloideus lateralis radii) base del IV metacarpiano (IV metacarpale), ligamento colateral lateral metacarpofalangeano (lig. collaterale metacarpophalangi laterale) y borde coronario (margo coronalis). En el miembro pelviano las marcas se emplazaron sobre la tuberosidad coxal (tuber coxae), trocánter mayor del fémur (trochanter major femoris (pars caudalis), ligamento rotuliano (lig. collaterale geni laterale) maléolo tibial lateral (malleolus lateralis tibialis), base del IV hueso metatarsiano (IV metatarsale), ligamento colateral lateral metatarsofalangeano (lig. collaterale metatarsophalangi laterale) y borde coronario del pié (margo coronalis) (Figura №1).

Antes de realizar la actividad a cada animal se los hizo hizo pasar por el sitio a recorrer durante 5 minutos. Seguidamente se les hizo recorrer dos veces una distancia de 15 metros al trote llevados de tiro, sobre piso de césped. En cada pasada se capturó una imagen del animal, del lado izquierdo, con una cámara de video digital de $60 \mathrm{~Hz}$ ubicada a 15 metros de forma perpendicular a los mismos. Los fotogramas obtenidos de los videos se introdujeron en el software ImageJ para determinar los ángulos de protracción-retracción de los miembros torácico y pelviano, de extensión y flexión máximas de los ángulos de inclinación escapular (AIE), de inclinación pelviana (AIP), de las articulaciones del hombro, codo, carpo y nudo del miembro torácico y de la cadera, rodilla, tarso y nudo del miembro pelviano.

El AIE se determinó haciendo pasar una línea imaginaria paralela al plano horizontal que pasó por la marca correspondiente a la tuberosidad del húmero para luego formar un ángulo con la marca correspondiente a la tuberosidad de la espina. El AIP se obtuvo de trazar una línea horizontal imaginaria que pasó por la marca correspondiente al trocánter mayor del fémur y se continuó con la marca de la tuberosidad coxal. El ángulo de protracción-retracción torácico se formó haciendo pasar una línea horizontal por la marca de la cruz y el marcador ubicado en el borde coronario del pié ${ }^{[14]}$ y el ángulo de 
protracción-retracción del miembro pelviano se tomó haciendo pasar una línea imaginaria horizontal por la marca del trocánter mayor del fémur que se continúa hacia la marca del borde coronario del pié ipsilateral (Figura №1). La amplitud angular (AA) se calculó de restarle al ángulo máximo en la extensión articular el valor del ángulo mínimo de flexión en cada articulación mencionada.
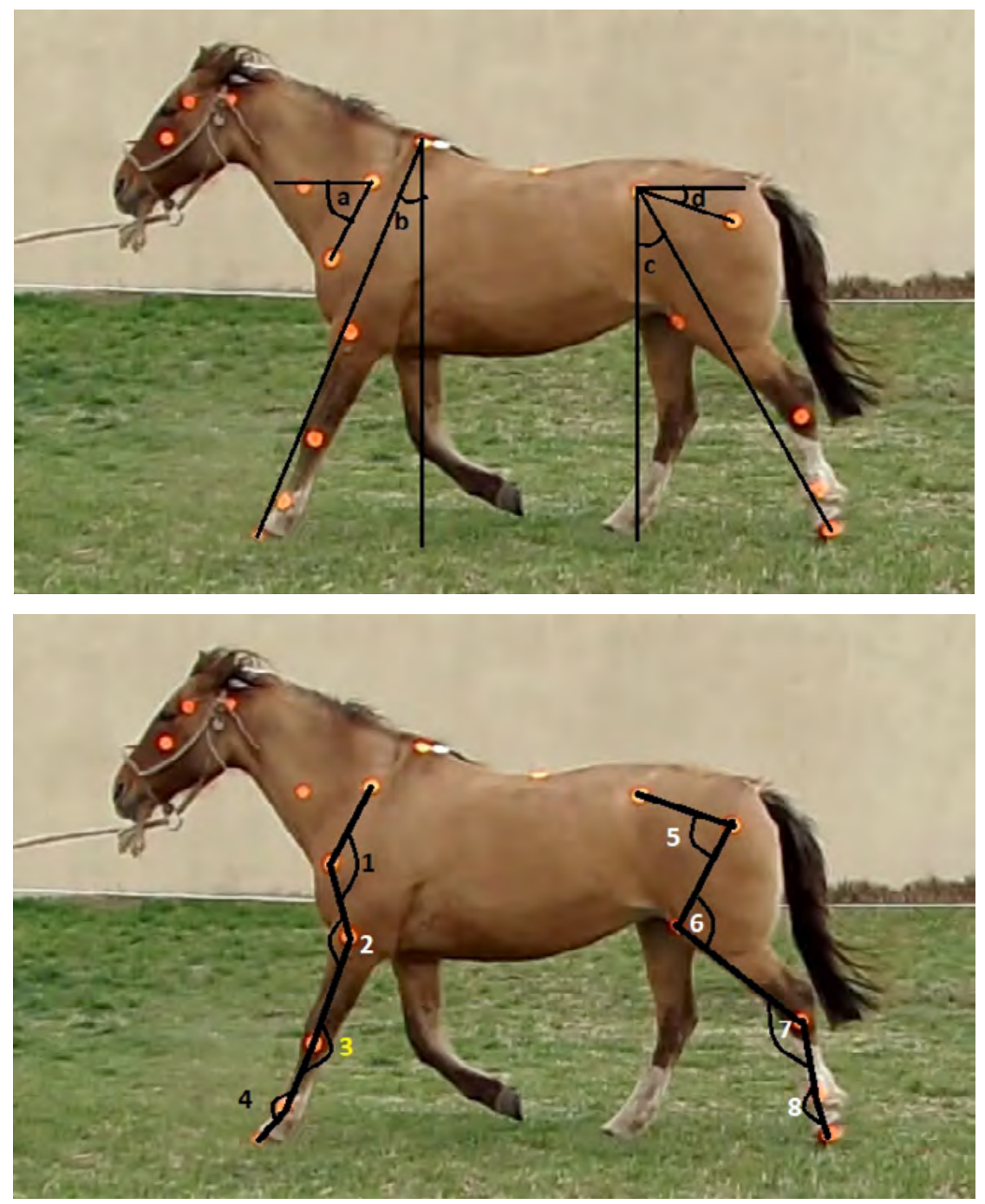

Figura №1. a) Ángulo de inclinación escapular, b) ángulo de protracción miembro torácico; c) ángulo de protracción del miembro pelviano; d) ángulo de inclinación pelviana; ángulos de las articulaciones del hombro (1), codo (2), carpo (3), articulación coxofemoral (5), rodilla (6), tarso (7) y nudos de los miembros torácicos y pelvianos (4 y 8). 
Los fotogramas fueron empleados para establecer la frecuencia de zancadas para el andar y establecer la cantidad de batidas (conjunto de zancadas que constituyen la unidad del andar) en una distancia de 15 metros.

La información obtenida se introdujo en el software Infostat (UNCCórdoba, Argentina ${ }^{[19]}$ a los efectos de realizar descripción estadística de la información registrada.

\section{RESULTADOS}

La altura media tomada a la cruz fue $157,45 \mathrm{~cm}$. $( \pm 7,44)$. Los caballos empleados fueron llevados de tiro al trote a una velocidad media de 4,08 m/seg. Los 15 metros de recorrido lo realizaron en 22,99 m/ seg., en ese espacio ejecutaron 5,69 zancadas. La longitud de cada batida fue 2,66 m. y demandó 0,65 seg. para ejecutarla (Tabla №1)

Tabla №1. Parámetros lineales de los Caballos Criollos Argentinos

\begin{tabular}{|c|c|c|c|c|c|} 
Caballo & $\begin{array}{c}\text { Velocidad } \\
\text { (m/seg) }\end{array}$ & $\begin{array}{c}\text { Velocidad } \\
\text { en } \mathbf{1 5} \mathbf{~ m}(\mathbf{m} / \\
\mathbf{s e g})\end{array}$ & $\begin{array}{c}\text { Longitud de } \\
\text { batida (m) }\end{array}$ & $\begin{array}{c}\text { Duración de } \\
\text { una batida } \\
\text { (seg) }\end{array}$ & $\begin{array}{c}\text { № de zanca- } \\
\text { das / 15 m }\end{array}$ \\
\hline 1 & 4,54 & 22,15 & 3,08 & 0,67 & 4,95 \\
\hline 2 & 3,26 & 19,48 & 2,51 & 0,77 & 5,98 \\
\hline 3 & 4,02 & 24,97 & 2,42 & 0,60 & 6,26 \\
\hline 4 & 3,92 & 22,50 & 2,61 & 0,66 & 5,77 \\
\hline 5 & 4,23 & 24,00 & 2,64 & 0,62 & 5,67 \\
\hline 6 & 5,15 & 25,82 & 3,00 & 0,58 & 5,01 \\
\hline 7 & 3,56 & 22,29 & 2,40 & 0,67 & 6,25 \\
\hline 8 & 3,99 & 22,77 & 2,63 & 0,66 & 5,70 \\
\hline MEDIAS & 4,08 & 22,99 & 2,66 & 0,65 & 5,69 \\
\hline
\end{tabular}

En conformidad a las variables lineales informadas, los ángulos de protracción y retracción, inclinación pélvica y escapular, extensión y flexión de las articulaciones de los miembros torácico y pelviano se encuentran expresados en la Tabla №2. 
Tabla №2. Variabilidad de los Ángulos de Extensión y Flexión de las Articulaciones de los Miembros del Caballo Criollo Argentino

\begin{tabular}{|l|c|c|c|}
\hline Miembro torácico & $\begin{array}{c}\text { Extensión } \\
\text { (grados) }\end{array}$ & $\begin{array}{c}\text { Flexión } \\
\text { (grados) }\end{array}$ & $\begin{array}{c}\text { Amplitud articular } \\
\text { (grados }\end{array}$ \\
\hline Protracción & 22,63 & & \\
\hline Retracción & 6,80 & & 12,4 \\
\hline AIE & 73,19 & 60,79 & 11,13 \\
\hline Hombro & 128,07 & 116,93 & 15,52 \\
\hline Codo & 159,85 & 144,60 & 3,66 \\
\hline Carpo & 175,85 & 172,18 & 32,79 \\
\hline Nudo MA & 159,68 & 126,89 & \\
\hline Miembro pelviano & & & 6,58 \\
\hline Protracción & 9,06 & & 17,18 \\
\hline Retracción & 22,03 & & 20,01 \\
\hline AIP & 33,11 & 26,52 & 10,94 \\
\hline Coxofemoral & 103,38 & 86,19 & 31,41 \\
\hline Rodilla & 138,57 & 118,56 & \\
\hline Tarso & 153,79 & 142,85 & \\
\hline Nudo MP & 161,09 & 129,68 & \\
\hline
\end{tabular}

Los ángulos de protracción y retracción del miembro torácico fueron $22,63^{\circ}$ y $6,8^{\circ}$; y 9,06 y $22,03^{\circ}$ para el miembro pelviano. El ángulo AIE mostró la máxima verticalidad de escápula en 73,19o y una inclinación de 60,79o con una variación de AA de 12,4‥ La media para el mismo ángulo se encontró en $67,35^{\circ}( \pm 4,46)$. El carpo fue la articulación del miembro torácico que presentó menor variación angular, entre una extensión de 175,85으 y flexión $172,18^{\circ}$ con una AA de 3,66으 y una media de $120,82^{\circ}( \pm 1,47)$. La articulación que mostró poseer mayor movimiento fue el nudo cuya AA fue 32,79 o proveniente de $159,68^{\circ}$ en extensión y 126,89 en la flexión y una media de $137,9^{\circ}( \pm 11,11)$. El hombro y codo presentaron AA similares, $11,13^{\circ}$ y $15,52^{\circ}$ respectivamente; en extensión alcanzaron 128,07으 y 159,85을 mientras que flexionaron hasta $116,93^{\circ}$ y $144,60^{\circ}$. Las medias de estas dos últimas articulaciones fueron $120,82^{\circ}( \pm 3,61)$ y $147,48^{\circ}\left( \pm 8,88^{2}\right)$ (Tabla №2) (Figura №2). 

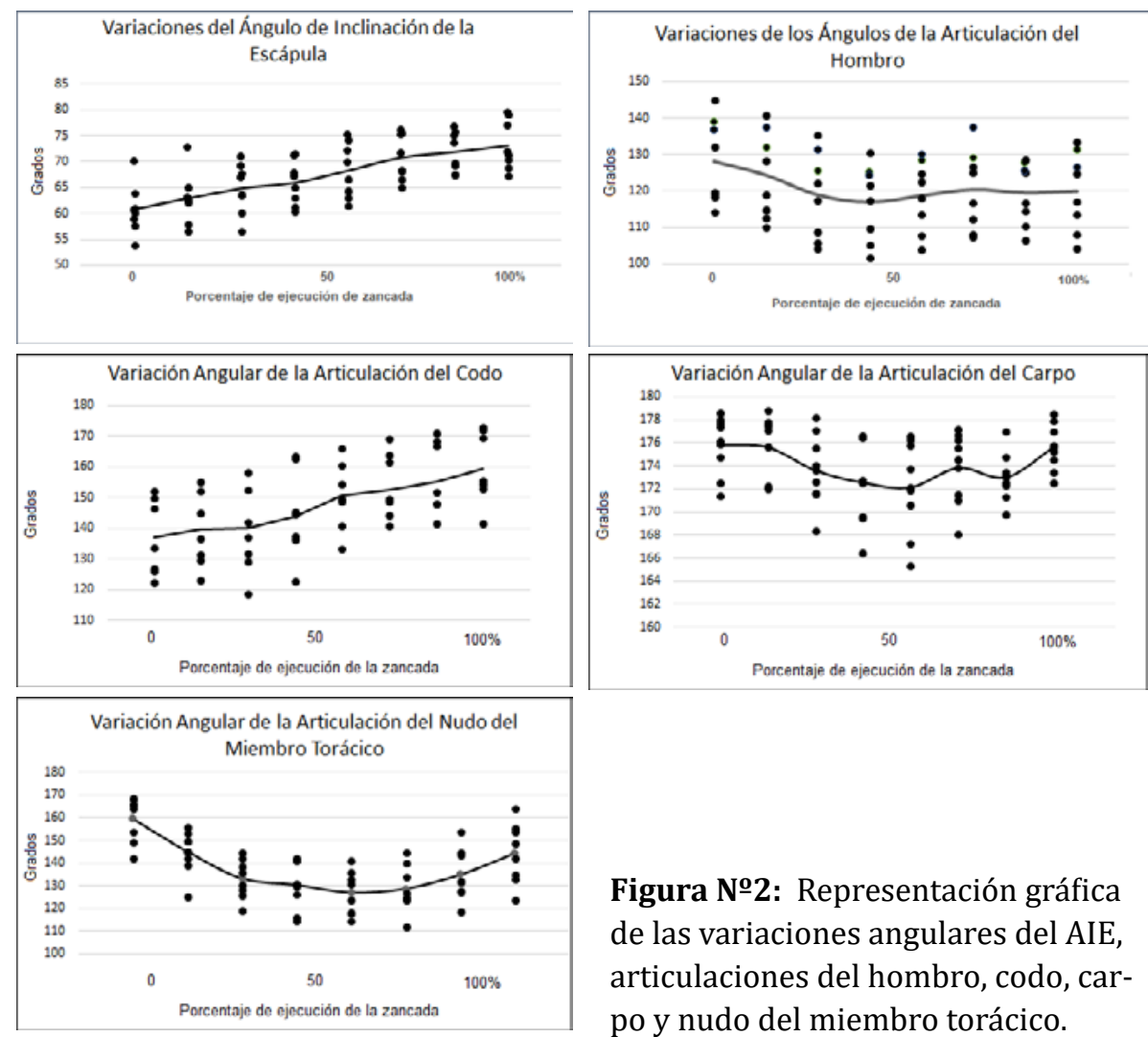

Figura №2: Representación gráfica de las variaciones angulares del AIE, articulaciones del hombro, codo, carpo y nudo del miembro torácico.

El AIP más vertical fue 33,11ำ mientras que la máxima flexión fue $26,52^{\circ}$ con una AA de 6,58․ La articulación de la cadera La articulación del tarso en el miembro pelviano fue la que mostró menor AA $\left(10,94^{\circ}\right)$, y la de mayor AA también fue el nudo. La articulación coxofemoral y la rodilla presentaron AA similares $\left(17,18^{\circ}\right.$ y $20,01^{\circ}$ respectivamente) (Tabla №2) (Figura №3). 

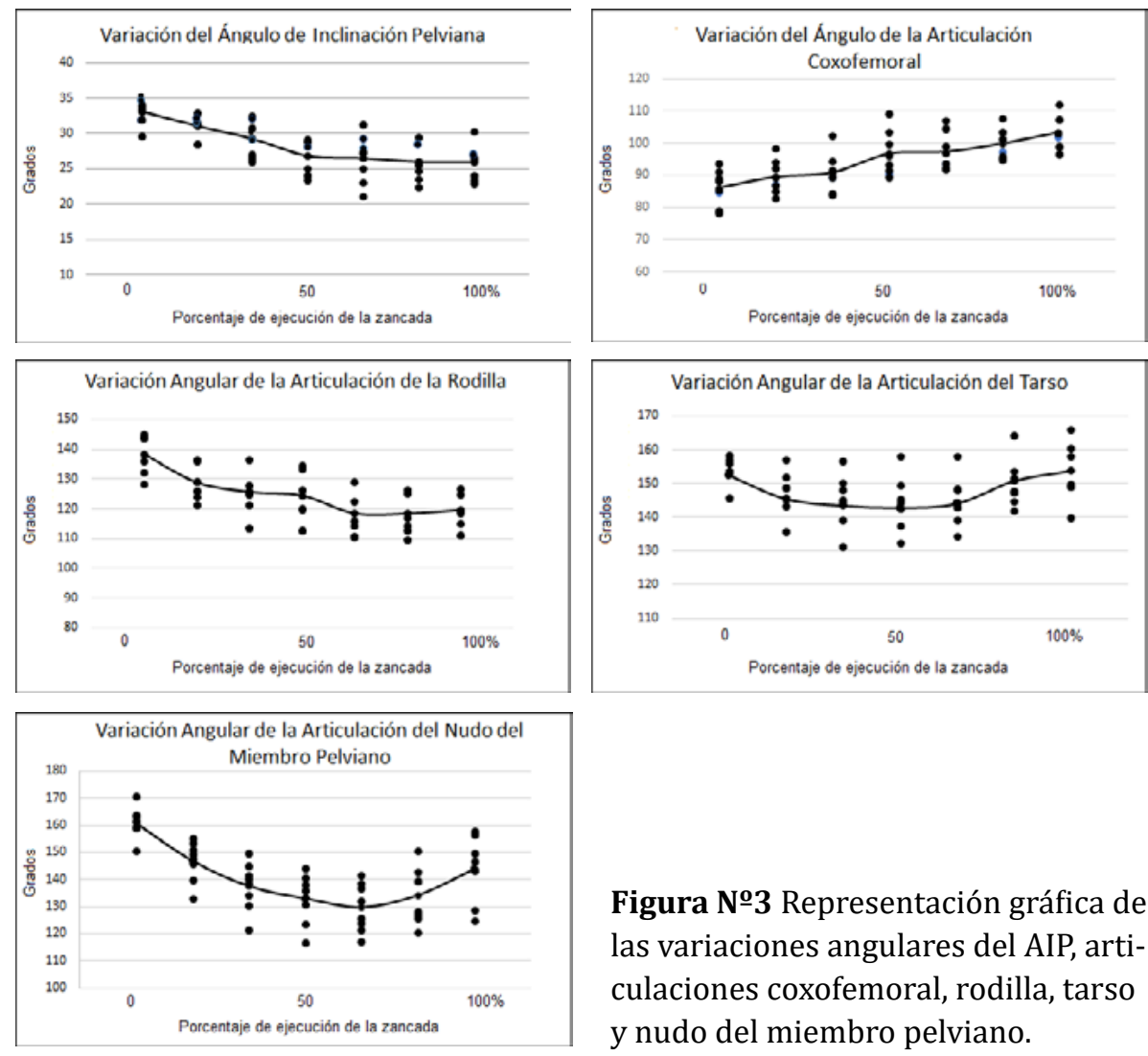

Figura №3 Representación gráfica de las variaciones angulares del AIP, articulaciones coxofemoral, rodilla, tarso y nudo del miembro pelviano.

Los cambios angulares de las articulaciones del hombro, codo, carpo, cadera, rodilla, tarso y menudillos de los miembros torácicos y pelvianos se exponen en la Tabla №3. 
Tabla No3: Variaciones angulares de las articulaciones estudiadas durante la ejecución de una zancada

\begin{tabular}{|c|c|c|c|c|c|c|c|c|c|c|}
\hline \multicolumn{9}{|c|}{ Miembro Torácico } \\
\hline Caballo & AIE & Hombro & Codo & Carpo & Nudo & AIP & $\begin{array}{c}\text { Coxo- } \\
\text { femoral }\end{array}$ & Rodilla & Tarso & Nudo \\
\hline 1 & 60,790 & 128,072 & 136,825 & 175,850 & 159,688 & 33,112 & 86,196 & 138,577 & 152,482 & 161,090 \\
\hline 2 & 62,907 & 124,322 & 139,124 & 175,642 & 145,075 & 31,103 & 89,515 & 129,053 & 145,472 & 146,970 \\
\hline 3 & 64,836 & 118,885 & 138,645 & 173,645 & 133,123 & 29,290 & 90,859 & 127,464 & 144,455 & 137,570 \\
\hline 4 & 65,973 & 116,936 & 144,600 & 172,576 & 130,301 & 28,080 & 93,815 & 125,876 & 143,438 & 132,974 \\
\hline 5 & 68,315 & 118,679 & 150,591 & 172,181 & 126,896 & 26,870 & 96,772 & 124,626 & 142,850 & 129,684 \\
\hline 6 & 70,856 & 120,333 & 153,790 & 173,853 & 128,678 & 26,526 & 97,325 & 118,649 & 144,044 & 134,229 \\
\hline 7 & 71,924 & 119,445 & 156,390 & 173,037 & 134,983 & 26,201 & 99,873 & 118,569 & 150,778 & 139,098 \\
\hline 8 & 73,192 & 119,924 & 159,857 & 175,638 & 144,461 & 25,994 & 103,984 & 119,780 & 153,794 & 143,967 \\
\hline Media & 67,35 & 120,82 & 147,48 & 174,05 & 137,90 & 28,42 & 94,84 & 125,02 & 147,55 & 140,93 \\
$( \pm 4,46)$ & $( \pm 3,61)$ & $( \pm 8,88)$ & $( \pm 1,47)$ & $( \pm 11,11)$ & $( \pm 2,82)$ & $( \pm 6,15)$ & $( \pm 7,19)$ & $( \pm 4,64)$ & $( \pm 10,79)$ \\
\hline
\end{tabular}

\section{Discusión}

En nuestro estudio los caballos se desenvolvieron con naturalidad y comodidad. Los valores de la experiencia no pueden ser comparados con experiencias similares en caballos Criollos Argentinos por no existir publicaciones que den cuenta de trabajos similares, ya sea en caballos llevados de la mano o sobre cintas mecánicas.

Los caballos guiados de la mano tienden a repetir las velocidades de un modo espontáneo y que se traduce en un patrón locomotor similar en pruebas sucesivas del mismo animal o durante comparaciones entre distintos individuos ${ }^{[3]}$. La baja variación de la velocidad registrada en pruebas individuales sucesivas y entre sujetos sugiere, de forma similar, a lo informado por Degueurce y col. ${ }^{[20]}$ que es posible repetir la experiencia sobre pista sin requerir la cinta de ejercicio.

Van Weeren y col. ${ }^{[21]}$ indicaron que el uso de marcadores refractantes de contacto para el estudio puede presentar errores provenientes de los movimientos que puede sufrir la piel en las referencias anatómicas, particularmente en las articulaciones proximales. Los mismos autores señalaron que los caballos de talla y conformación similares tienen movimientos de la piel de magnitud comparable. Coincidiendo con Miró Rodríguez y col. ${ }^{[22]}$ los autores concordamos en que individuos de una misma raza, de conformación semejante y de talla parecida, los resultados obtenidos para estas variables podrían, con las 
debidas precauciones, ser tenidos en cuenta como referencia de esta raza.

Entre caballos de la misma raza existe correlación positiva entre la altura a la cruz y las variables lineales como lo es la longitud de zancada o de tranco ${ }^{[3]}$.

En el caballo Criollo Argentino al trote llevado de la mano hallamos que el AIE fue 73,19o en el momento de mayor extensión y 60,79응 en la flexión con una AA de 12,4‥ Estudios realizados en condiciones similares señalan que caballos de las razas Anglo-Árabes poseen una AA del AIE es 18,7을 en Árabes $21,2^{\circ}$; Andaluces $20,4^{\circ}$ y en Dutch Warmbloods la misma AA fue $18,5^{\circ}[3,17]$.

El AIE indica el grado de inclinación que alcanza la escápula durante la ejecución de la zancada. Una escápula que alcanza una posición de mayor horizontalidad induce al miembro anterior a pendular a una posición de mayor protracción, es decir lleva al casco más hacia adelante. Así, el AIE se relaciona con el nivel de performance del caballo ${ }^{[23]}$. Por otra parte, una escápula que tiende a la verticalidad predispone a contusiones en el momento de apoyo del pie en la zancada ${ }^{[24]}$.

La protracción está estrechamente relacionada con propiedades pasivas de las extremidades para el avance en lugar del trabajo muscular ${ }^{[25,26]}$, aunque algo de acción muscular se requiere para hacer pivotar cada miembro hacia adelante para iniciar el siguiente paso o zancada $^{[27]}$. Los caballos no pueden alcanzar la energía requerida para alcanzar la potencia necesaria para la protracción rápida sólo con la contracción muscular ${ }^{[26]}$

Establecer los rangos de retracción-protracción de los miembros torácico y pelviano contribuye a conocer la sincronicidad de la retracción ipsilateral de la extremidad anterior y la protracción del miembro posterior en el trote, pues se encuentra ligada a una mayor flexibilidad en la flexión lateral del cuerpo que se observa en caballos en la marcha natural ${ }^{[28]}$.

Valera ${ }^{[8]}$ y Molina ${ }^{[29]}$ establecieron las variables biocinemáticas para ser empleadas en programas de selección y reproducción con fines de rendimiento deportivo y de trabajo. Entre esas variables, se incluye el rango máximo de retracción-protracción. El conocimiento del rango de los ángulos de retracción-protracción del caballo Criollo Argentino aporta información de esa variable para programas de cría con el objeto de obtener animales de mejor performance.

Para que el miembro torácico alcance la mayor protracción, simultáneamente se produce la flexión del hombro con máxima extensión del codo al momento en que el casco hace contacto con el suelo ${ }^{[1,29]}$. Igual que en la protracción, para que se efectúe la retracción se produce la 
máxima extensión del hombro, codo y carpo, aunque la inclinación de la escápula adquiere mayor verticalidad antes de que el casco despegue del suelo.

En referencia a las articulaciones del nudo no se apreciaron diferencias sustanciales entre los ángulos de extensión, flexión y AA. En ambos, una mayor extensión articular se relaciona con mayor protracción. Las diferencias entre ambas articulaciones son de carácter biomecánicas, ya que el nudo del miembro torácico posee un rol de flexibilidad mientras que el nudo de la extremidad pelviana interviene mecánicamente en el impulso del caballo ${ }^{[1,30]}$.

La flexibilidad de la articulación coxofemoral a través de la AA es debido a que esta se corresponde con mayor movimiento de la articulación lumbosacra y por consiguiente transfiere mayor flexibilidad a los lomos. Esta interacción mecánica es esencial para transmitir el impulso generado por los miembros pelvianos a los miembros torácicos ${ }^{[31]}$.

El tarso, al igual que el carpo, mostró mayor extensión articular en los momentos de apoyo y despegue del suelo del casco, que son los momentos de mayor protracción y retracción del miembro pelviano.

\section{Conclusiones}

Los autores del presente estudio establecimos las variaciones angulares de las articulaciones de los miembros en caballos criollos Argentinos asociados al trote cuando es llevado de la mano. Se requieren nuevos y más estudios para establecer las angulaciones en otros andares como el paso y realizar comparaciones. No obstante, nuestro estudio aporta nuevos datos que podrían contribuir a la exploración física de claudicaciones y como referencia para evaluar la evolución de tratamiento de cojeras. 


\section{Bibliografía}

1. Buchner HH, Savelberg HH Schamhardt HC, Barneveld A. Head and trunk movement adaptations in horses with experimentally induced foreor hindlimb lameness. Equine Vet J.1996; 28:71-76.

2. Buchner, HH, Savelberg HH, Schamhardt HC, Barneveld A. Limb movement adaptations in horses with experimentally induced fore- or hindlimb lameness. Equine Vet. J. 1996b; 28, 63-70

3. Galisteo AM, Vivo J, Cano MR, Morales JL, Miró F, Agüera E. Differences between breeds Dutch Warmblood vs Andalusian Purebreed in forelimb kinematics. J Equine Sc. 1998; 8:43-47.

4. Clayton HM, Schamhardt HC, Willemen MA, Lanovaz JL, Colborne GR. Kinematics and ground reaction forces in horses with superficial digital flexor tendinitis. Am J Vet Res. 2000; 61:191-196.

5. Kramer J, Keegan KG, Wilson DA, Smith BK, Wilson DJ. Kinematics of the hind limb in trotting horses after induced lameness of the distal intertarsal and tarsometatarsal joints and intra-articular administration of anesthetic. Am J Vet Res.2000 ; 61:1031-1036.

6. Buchner HH, Obermuller S, Scheidl M. Body centre of mass movement in the lame horse. Equine Vet J Suppl. 2001; 33:122-127.

7. Valera M, Galisteo AM, Molina A, Miro F, Gómez MD, Cano MR, Agüera E. Genetic parameters of biokinematic variables of the trot in Spanish Purebred horses under experimental treadmill conditions. 2008; Vet J. 178:219-26.

8. Buchner HH, Savelberg HCM. Inertial properties of Dutch Warmblood horses. J Biomech. 1997 ; 30:653-658.

9. Clayton HM, Sha, D, Stick JA, Mullineaux DR. Three-dimensional carpal kinematics of trotting horses. Equine Vet J.2004; 36:671-676

10. Robert C, Valette JP, Pourcelot P, Audigié F, Denoix JM. Effects of trotting speed on muscle activity and kinematics in saddlehorses. Eq Vet J Suppl. 2005 ; 34:295-301

11. Galloux P, Barrey E. Components of the total kinetic moment in jumping horses. Eq Vet J Suppl.1997; 23:41-44.

12. Swanstrom MD, Zarucco L, Hubbard M. Musculoskeletal modeling and dynamic simulation of the thoroughbred equine forelimb during stance phase of the gallop. J Biomech Eng. 2005 ; 127:318-328.

13. Valette JP, Barrey E, Auvinet B, Galloux P, Wolter R. Comparison of track and treadmill exercise tests in saddle horses: a preliminary report. Annale de Zootechnie. 1992 ; 41:129-135.

14. Escobar A, Tadich T. Caracterización biocinemática, al paso guiado a la mano, del caballo fino chilote. Arch Med Vet. 2006; 38:53-61.

15. Martuzzi F, Vaccari Simonini F, Gosi S, Catalano AL. Kinematic characteristics of walk in the Bardigiano Horse breed caratteristiche della cinematica del passo nella razza cavallo Bardigiano. Ann Fac Medic Vet di Parma. 2007; 27:229- 234. 
16. Solé M, Gómez MD, Galisteo AM, Rute S, Valera M. Kinematic Characterization of the Menorca Horse at the Walk and the Trot: Influence of Hind Limb. J Eq Vet Sc. 2013 ; 33:726-732.

17. Cano MR, Vivo J, Miró F, Morales JL, Galisteo AM. Kinematic characteristics of Andalusian, Arabian and Anglo-Arabian horses: a comparative study. Res Vet Sc. 2001 ; 71:147-153.

18. Zips S, Peham C, Scheidl M, Licka T, Girtler D. Motion pattern of the toelt of Icelandic horses at different speeds. Equine Vet. J. 2001 ; S33:109-111.

19. Di Rienzo JA, Casanoves F, Balzarini MG, Gonzalez L, Tablada M, Robledo CW. InfoStat versión .2010 Grupo InfoStat, FCA, Universidad Nacional de Córdoba, Argentina

20. Degueurce C, Pourcelot P, Audigie F, Denoix JM, Geiger D. Variability of the limb joint patterns of sound horses at trot. Equine Vete J Suppl. 1997 ; 23:89-92.

21. Van Weeren PR, van Bogert AJ, van den Barneveld A. Correction models for skin displacement in equine kinematic gait analysis. J Equ Vet Sc. 1992 ; 12:178-192

22. Miró Rodríguez F, Martínez Galisteo A, Vivo Rodríguez J, Cano García R. Patrón locomotor del trote del caballo Pura Raza Español y su variabilidad. Rev Elec Clínica Vet. $2008 ; 3: 1-17$.

23. Holmström M, Magnusson LE, Philipsson J. Variation in conformation of Swedish warmblood horses and conformational characteristics of elite sport horses. Equine Vet J. $1990 ; 22: 186-193$.

24. Holmström M, Philpsson J. Relationships between conformation, performance and health in 4-year-old Swedish Warmblood riding horses. Livestock Prod Sc.1993; 33:293-312.

25. Heglund NC, Cavagna GA, Taylor CR. Energetics and mechanics of terrestrial locomotion. III. Energy changes of the centre of mass as a function of speed and body size in birds and mammals. J Exp Biol. 1982 ; 79:41-56.

26. 26 Wilson AM, Watson JC, Lichtwark GA. Biomechanics: a catapult action for rapid limb protraction. Nature. 2003 ; 421:35-36.

27. $27 \mathrm{Kram}, \mathrm{R}$. Muscular force or work: what determines the metabolic energy cost of running? Exerc. Sport Sci. Rev. $2000 ; 28,138-143$.

28. 28 Back W, Schamhardt HC, Savelberg HHCM, Van Den Bogert AJ, Bruin G Hartman W. How the horse moves: 2. Significance of graphical representations of equine hind limb kinematics. Equine Vet J. 1994 ; 27:39-45.

29. 29 Molina A, Valera M, Galisteo AM, Vivo J, Gómez MD, Rodero A, Agüerac E. Genetic parameters of biokinematic variables at walk in the Spanish Purebred (Andalusian) horse using experimental treadmill records. Livestock Sc. 2008; 116(1-3):137-145.

30. Back W, Schamhardt HC, Barneveld A. Are kinematics of the walk related to the locomotion of a warmblood horse at the trot? Vet Quarterly. 1996 ; 18:79-84.

31. Bennett D. 1992. Principles of conformation analysis. Volume I. Fleet street Publishing Co. Gaithesburg. USA. 
